Thermische und chemische

Verletzungen

\section{Lokalanästhesie mit Adre- nalinzusatz bei der Lösung von Flexionskontrakturen nach Verbrennungen}

Bashir MM et al. Outcomes of post burn flexion contracture release under tourniquet versus tumescent technique in children. Burns 2018; 44 : $678-682$

Flexionskontrakturen sind nach Verbrennungen v.a. bei Kindern eine häufige Komplikation. Meist erfolgen die Lösung dieser Kontrakturen und die Deckung des Defekts mit einem Hauttransplantat in Blutleere unter Allgemeinanästhesie.

Die Blutleere wird dabei für eine ausreichende Übersicht über das Operationsfeld als unabdingbar betrachtet - aber vielleicht kann dieser Effekt auch auf andere Weise erreicht werden. Mediziner aus Pakistan haben das untersucht.

Muhammad Bashir und seine Kollegen haben dazu insgesamt 160 Kinder im Alter zwischen 3 und 12 Jahren in eine randomisierte Studie aufgenommen. Einschlusskriterium war eine Flexionskontraktur nach Verbrennung im palmaren Bereich von Hand und/oder Fingern, bei der nach Behebung ein Hauttransplantat mit einer Größe $>2 \mathrm{~cm}^{2}$ erforderlich war. Kinder mit Rezidivkontrakturen waren allerdings ausgeschlossen.

Jeweils 80 Patienten wurden nach dem Zufallsprinzip 1 von 2 Behandlungsgruppen zugewiesen:

- klassische Operation in Allgemeinanästhesie und Blutleere $(100 \mathrm{mmHg}$ oberhalb des systolischen Blutdrucks) (Gruppe 1)

- Operation in Allgemeinanästhesie und zusätzlicher lokaler Injektion einer Tumeszenzanästhesie-Lösung (pro $100 \mathrm{ml}$ physiologische Kochsalzlösung Zugabe von 200 mg Lidocain $2 \%$ plus $1 \mathrm{ml}$ Adrenalin 1:1000, entsprechend einer endgültigen Lidocain-Konzentration von $0,18 \%$ und einer Adrenalin-Konzentration von 1:111000) (Gruppe 2)

In Gruppe 1 begann die Operation unmittelbar nach Erreichen der Blutleere, in Gruppe 225 min nach Einbringen der Tumeszenz-Lösung, wenn die Haut straff gespannt und blass geworden war.

Als Endpunkte beurteilten die Mediziner die Operationsdauer, die postoperative Schmerzstärke anhand des Analgetikabedarfs und des FLACC-Scores (FLACC: Face, Legs, Activity, Cry, Consolability; eine in der Pädiatrie häufig genutzte Skala zur Messung der Schmerzintensität) und das Einheilen des Hauttransplantats bis Tag 14 nach dem Eingriff. Das Durchschnittsalter der Kinder betrug knapp 8 Jahre, Jungen machten gut die Hälfte der Gruppe aus (52,5\%). Die Auswertung ergab

- eine vergleichbare Operationsdauer in den beiden Gruppen $(67,7 \mathrm{~min}$ in Gruppe 2 vs. 67,0 min in Gruppe 1), trotz der 25-minütigen Wartedauer bis zum OP-Beginn nach Injektion der Tumeszenz-Lösung,

- eine geringere Schmerzstärke in den ersten $24 \mathrm{~h}$ postoperativ in Gruppe 2, mit

- einem durchschnittlichen Nalbuphin-Bedarf von $6,3 \mathrm{mg}$ gegenüber 9,4 mg in Gruppe 1 und

- signifikant geringeren Punktwerten auf der FLACC-Skala (entsprechend einer geringeren Schmerzstärke) sowohl $6 \mathrm{~h}, 12 \mathrm{~h}$ als auch $24 \mathrm{~h}$ nach der Operation, sowie

- ein großflächigeres Einheilen des Hauttransplantats bis Tag 14 in Gruppe $2\left(8,97 \mathrm{~cm}^{2}\right.$ vs. $7,26 \mathrm{~cm}^{2}$ in Gruppe 1).

Wesentliche Komplikationen traten nicht auf. Insbesondere war in Gruppe 2 keine zweite Injektion von Tumeszenz-Lösung notwendig, und in keinem Fall musste wegen einer blutungsbedingten schlechten Übersicht im Operationsgebiet sekundär auf eine OP in Blutleere übergegangen werden.
FAZIT

Demnach könnte die Tumeszenz-

Technik (Lokalanästhesie mit Adrenalinzusatz) bei der Lösung von Flexionskontrakturen nach Verbrennungen im Kindesalter eine Alternative zur klassischen OP-Technik in Blutleere darstellen, so die Autoren. Die damit anscheinend geringere postoperative Schmerzstärke wirkt sich bei der anschließend notwendigen Physiotherapie möglicherweise besonders positiv aus. Allerdings müssen umfangreichere Studien, die auch eine längere Nachbeobachtungszeit über Tag 14 hinaus einschließen, diese Ergebnisse bestätigen.

Dr. Elke Ruchalla, Bad Dürrheim 